\title{
A fully encapsulated piezoelectric-triboelectric hybrid nanogenerator for energy harvesting from biomechanical and environmental sources
}

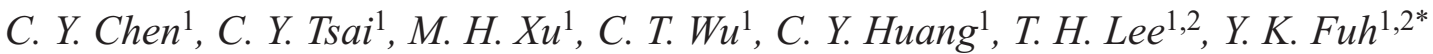 \\ ${ }^{1}$ Department of Mechanical Engineering, National Central University, Taoyuan City, Taiwan \\ ${ }^{2}$ Institute of Energy Engineering, National Central University, Taoyuan City, Taiwan
}

\begin{abstract}
In this paper, a hybrid nanogenerator with concurrently harvested piezoelectric and triboelectric mechanisms, called a fully encapsulated piezoelectric-triboelectric hybrid nanogenerator (PTHG), is demonstrated. In the construction of piezoelectric nanogenerator (PENG), an in-situ poling near-field electrospinning (NFES) was utilized to direct-write piezoelectric polymeric nano/micro fibers (NMFs) polyvinylidene fluoride (PVDF) as the functional layer of piezoelectric nanogenerators. On the other hand, the nano-textured functional layer of triboelectric nanogenerators (TENGs) is also concurrently combined with PENG. This hybridized nanogenerator was demonstrated to simultaneously harvest piezoelectric and triboelectric output such that the superimposed peak output voltage /current signals of $\sim 130 \mathrm{~V} / 4 \mu \mathrm{A}$ at $2 \mathrm{~Hz}$, which can be translated to the area power density of $8.34 \mathrm{~mW} / \mathrm{m}^{2}$. Individually measured TENG under a hand-induced strain 0.2 and $2 \mathrm{~Hz}$ actuation, the output voltage/current peak is measured about $110 \mathrm{~V} / 2.8 \mu \mathrm{A}$, while the PENG counterpart shows the the output voltage/current peak is about $18 \mathrm{~V} / 0.6 \mu \mathrm{A}$. In addition, the proposed PTHG can harvest sustainable energy sources such as rain water with the output maximum voltage reaches $\sim 20 \mathrm{~V}$ and area power density $\sim 0.981 \mathrm{~mW} / \mathrm{m}^{2}$ for dropping rate of $10 \mathrm{ml} / \mathrm{s}$. This research shows the substantial improvement in the synergy of nano-textured triboelectric and piezoelectric functional layers. The practical application of the self-powered system can be ubiquitously implemented in the sustainable energy sources and future industry 4.0 scenarios to provide the stand alone energy sources of IoT sensors.
\end{abstract}

Keywords: nanomaterials, $P T H G, P V D F, N M F$, NFES

\section{Introduction}

Owing to rapidly depleted fossil fuels with increasingly hazardous emission (particularly particulate matter PM2.5) over the past decades [1], significantly intensive research of renewable or green energy been adopted to ameliorate the imminent energy issues $[2,3]$. In particular, harvesting energy from sustainable resources, particularly the environmentally available mechanical vibration $[3,4]$ such as oceanic motion [5]. Furthermore, research thrust has been turned into the tiny human-induced motions operated in the manner of self-powered to differentially detect various biomechanical movement such as muscular motion of walking step [6-10].

Concerning the fundamental principles for the energy harvesting of mechanical-to-electric conversion mechanisms, different energy transduction mechanisms had been investigated such as electrowetting [11], electromagnetics [12], piezoelectricity [11-14], and triboelectricity $[15,16]$. The practical applications concentrated on the development of in nextgeneration flexible electronics and self-powered systems for motion monitoring sensors, mobile electronics and implanted medical devices [17-19].

\footnotetext{
${ }^{*}$ Corresponding author, e-mail: michaelfuh@gmail.com (C) BME-PT
} 
Piezoelectric materials have been widely investigated as the main energy transduction passages to effectively harvest energy from mechanical sources [20]. Piezoelectric nanogenerator (PENG) was previously focused on the 1D zinc oxide nanowires [3,21], polyvinylidene fluoride (PVDF) nanofibers [22]. Moreover, near-field electrospinning technique (NFES) [23] demonstrated that in-situ (mechanically and electrically) poled and highly aligned fibers can be fabricated directly on flexible substrates [24, 25].

On the other hand, recently developed triboelectric nanogenerator (TENG) also demonstrated the favorable capability to harvest various mechanical energies (vibrations [26-28], airflow [29], water waves $[30,31]$, rotation [4] and even acoustic energy [32]) in a sustainable manner [33, 34], TENG self-powered and sustainable harvesting sources can be applied to personal devices with light weight and simple fabrication [35-38].

In order to enhance the energy output and scale up both PENG or TENG, piezoelectric/triboelectric hybridized nanogenerator was develpoed successfully for finger-induced smart windows [39]. In addition, the Graphene-based Hybridized Self-Powered Sensor (GHSPS) demonstrated highly transparent and good flexible properties [40]. Another type of hybrid nanogenerators (TENG and electromagnetic nanogenerator) has been developed $[15,41,42]$ and furthermore, water flow-based blue energy from the environment [43]. Furthermore, some recent relevant research works related to triboelectric nanogenerators are also reviewed with distinctive features such as humidity-resisting triboelectric nanogenerator (HRTENG) [44], nanofibrous membrane constructed wearable triboelectric nanogenerator (NM-TENG) [45], functionalized charge-transport/charge-storage layer as multilayered fiber-based TENG [46] and a flexible self-charging power system by integrating a fiber-based supercapacitor and TENG [47].

This work represents a piezoelectrically and triboelectrically hybridized synergy and full encapsulation of PTHG such that the practical applications toward the sustainable power source for wearable electronics has been demonstrated.

\section{Experimental results}

Figure 1a illustrates a design concept and functional construction of a PTHG, where the $\sim 0.3 \mu \mathrm{m}$ thickness Zn-film (DJ-0130-45024 Taihokohzai Co., Ltd) based PENGs were fabricated on the top, and $\mathrm{Zn}$ film were deposited on the top of $400 \mu \mathrm{m}$ thickness Printed circuit board (PCB) light guide plate (LGP, Forhouse Corp.) with patterned microscale surface structures, and the piezoelectric NMFs were deposited on the $\mathrm{Zn}$-film. At last, LGP assembled together with a $80 \mu \mathrm{m}$ thickness $\mathrm{Cu}$ tape (ECTP50 MISUMI
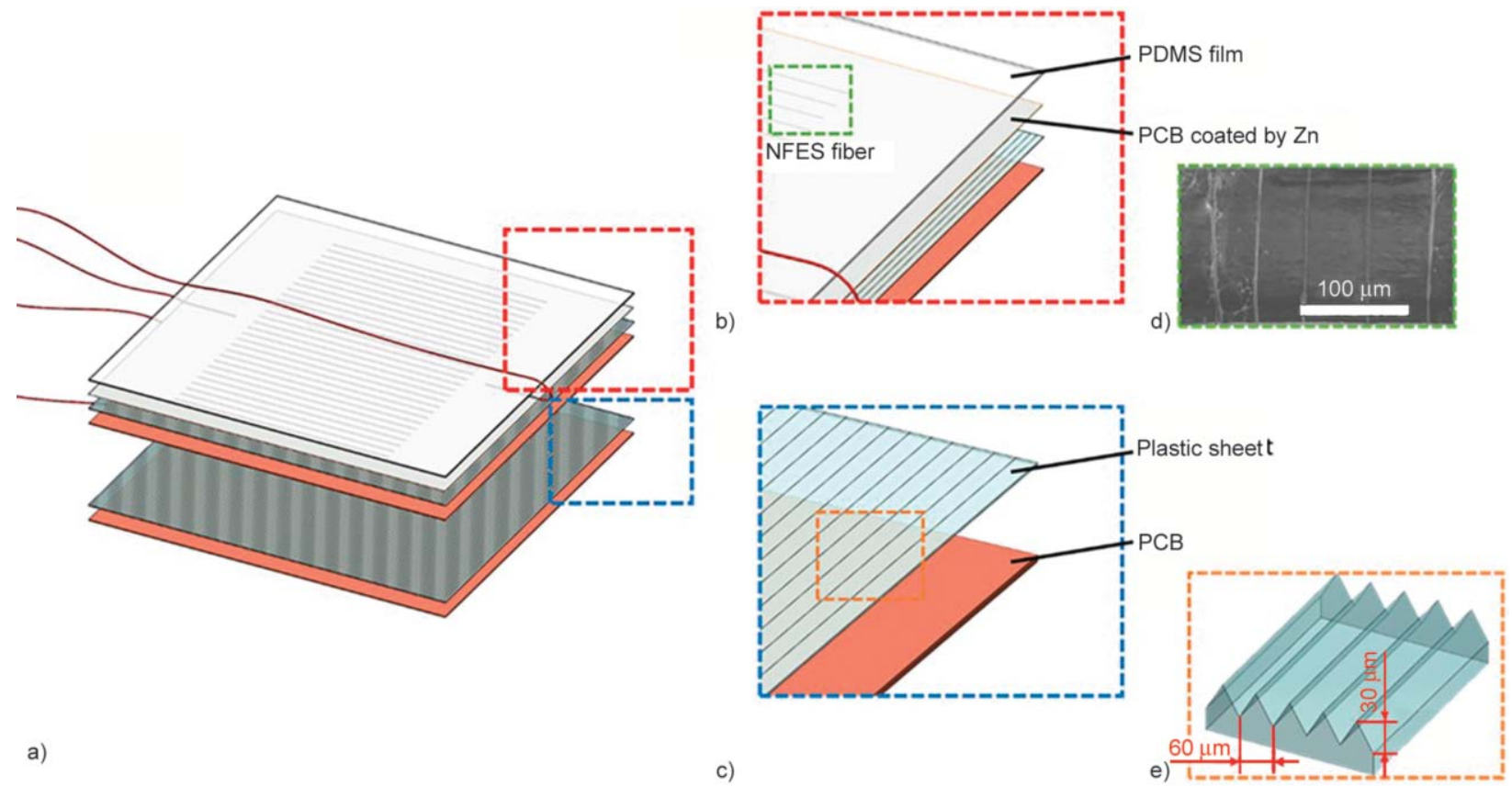

a)

c) e)

Figure 1. Schematic construction of the PTHG. (a) two functional components of PTHG, an upper layer (actuated piezoelectrically and triboelectrically) and a beneath layer (actuated only triboelectrically). A separate magnified image of (b) an upper layer and (c) a beneath layer. (d) A SEM image of the arrays of NFES fibers deposited on the Zn-film. (e) A surface pattern of light guide plate. 
Table 1. Detailed information regarding the typical setup of experiments procedure used to make the NFES electrospun NMFs.

\begin{tabular}{|l|c|}
\hline \multicolumn{2}{|c|}{ Near field electrospun nano/micro fibers } \\
\hline Applied voltage & $1.5 \mathrm{kV}$ \\
\hline Stage speed & $60 \mathrm{~mm} / \mathrm{s}$ \\
\hline Needle diameter & $0.17 \mathrm{~mm}$ \\
\hline Tip-to-substrate distance & $2.0 \mathrm{~mm}$ \\
\hline Syringe pump feed speed & $8 \cdot 10^{-5} \mathrm{ml} / \mathrm{s}$ \\
\hline Electric field & $\sim 10^{6} \mathrm{~V} / \mathrm{m}$ \\
\hline
\end{tabular}

Inc.) which work as a part of Cu-PCB TENG as friction parts in Figure 1b ( 1000 NMFs). On the other side, the LGP was assembled together with a $\mathrm{Cu}$ tape which work as a part of $\mathrm{Cu}-\mathrm{PCB}$ TENG as friction parts in Figure 1c. Furthermore, Cu-PCB TENG is an elastomer film. The Scanning Electron Microscope (SEM) morphology of the arrays of NFES fibers as shown in Figure 1d corresponds to the green dotted rectangle in Figure 1b. In addition, the shape designed in Figure 1e aims to simultaneously harvest and enhance the piezoelectric and triboelectric, which corresponds to the orange dotted rectangle in Figure 1c. Furthermore, the detailed specifications of NFES electrospun NMFs are illustrated in Table 1.

We used three different LGPs, which have different topologies as shown in Figure 2 and denoted as follows: planar surface, irregular protrusions surface (top surface of LGP), and triangular surface (bottom surface of LGP) are respectively shown in Figure 2a-2c. All samples have a dimension of $210 \mathrm{~mm} \times$ $297 \mathrm{~mm} \times 1 \mathrm{~mm}$ and the surface optical micrograph is displayed in Figure 2d-2f. For irregular protrusions surface, the height and pitch of protrusions are irregular, the highest height of protrusions are about $50 \mu \mathrm{m}$. For the triangular surface, the height is $30 \mu \mathrm{m}$ and pitch is $60 \mu \mathrm{m}$. An available direct current motor (RS-545SH) was utilized for cyclic stretching-releasing deformation, which experimentally operated at a strain of $0.5 \%$ and $2 \mathrm{~Hz}$. The output voltage was detected by an oscilloscope (Agilent DSO1014A). In Figure $2 \mathrm{~g}-2 \mathrm{i}$, the three different PTHG devices (planar, irregular protrusions, and triangular surface) can generate output voltages of about 40,58 , and $77 \mathrm{~V}$, respectively. From the experimental results, the device with triangular surface had the best output due to the longest fiber length, which was electrospun by NFES process and had the higher strain exerted on the substrate.

Figure $3 \mathrm{a}$ shows the optical photo of working condition of PTHG in the sequence of the original, press and release states, respectively (from left to right). Fundamentally, as the PTHG subjecting to the mechanical deformation such as hand-induced strain,

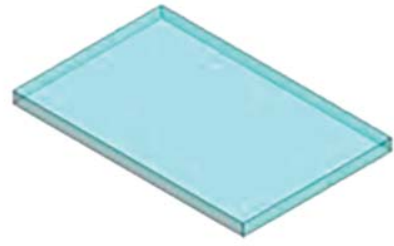

a)
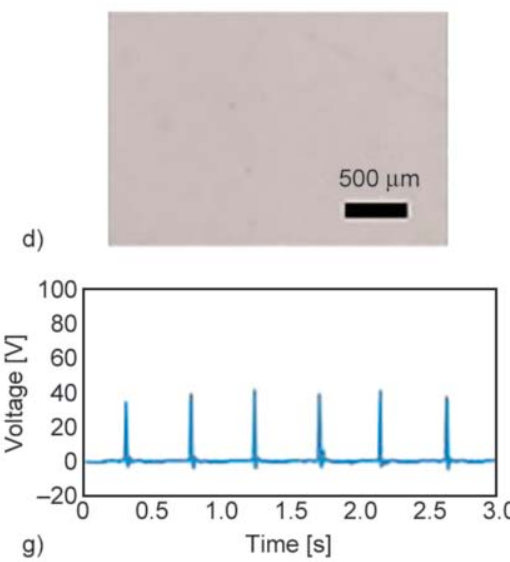

b)
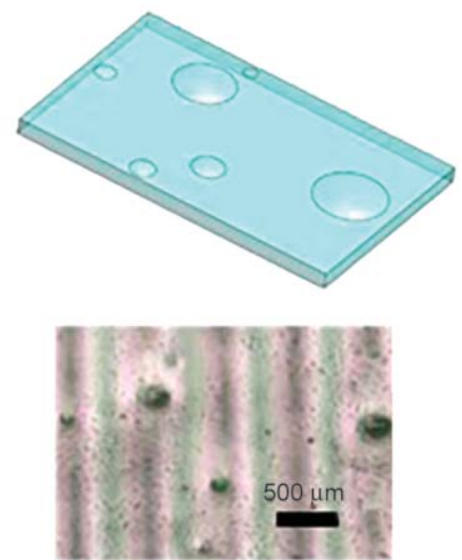

e)

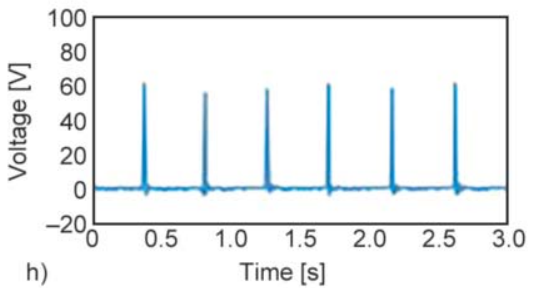

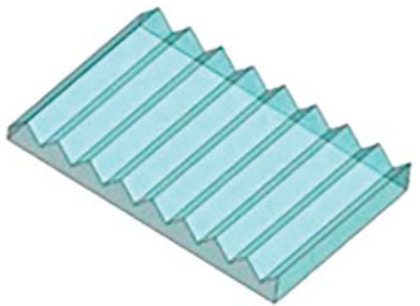
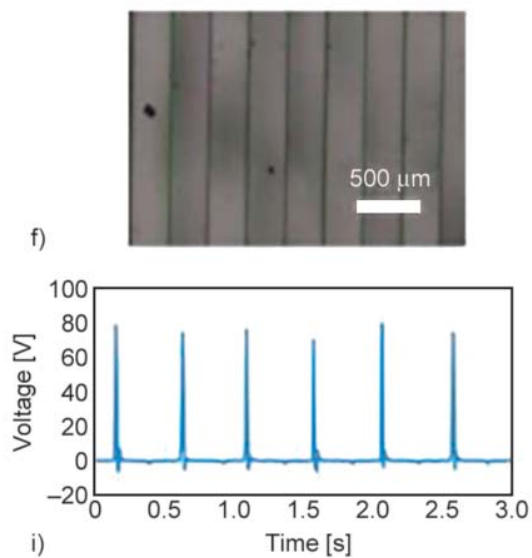

Figure 2. Schematic of three topologically different surfaces were investigated as: (a) planar surface, (b) irregular protrusions surface and (c) triangular surface. From (d-f) are the optical microscope image of three different surfaces topology respectively. From $(\mathrm{g}-\mathrm{i})$, the topologically tailored devices can stably produce about average output voltage $40 \mathrm{~V} / 58 \mathrm{~V} / 77 \mathrm{~V}$ respectively during the cyclic deformation. 
both piezo-potential (NMFs) and tribo-potential are concurrently generated by the physical strain as well as contact of $\mathrm{Cu}-\mathrm{PCB}$ interfaces. Figure $3 \mathrm{~b}$ depicts the TENG working principle by initially establishing the potential drop and physical contact between the elastomer film and the ground plate. During one motion cycle, two peak electrical output would be generated in the external circuit due to tribo-charge density and the output current should be dominated by the separation velocity $[48,49]$. The TENG measured output voltage signals of under a hand-induced strain 0.2 and $2 \mathrm{~Hz}$ actuation, the output voltage/current peak is measured at $\sim 110 \mathrm{~V} / 2.8 \mu \mathrm{A}$. Figure $3 \mathrm{c}$ presents the operating principle of PENG under the same mechanical-induced strain, the original generated piezoelectric dipoles can be collected through the
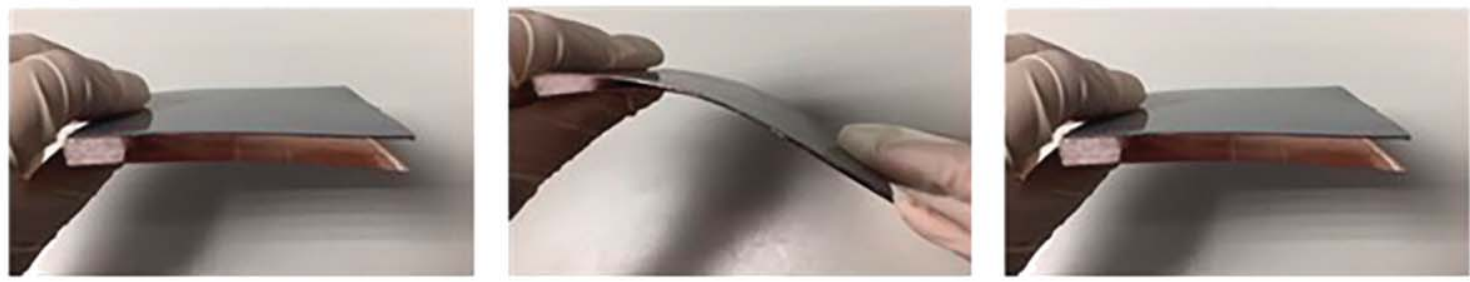

a)
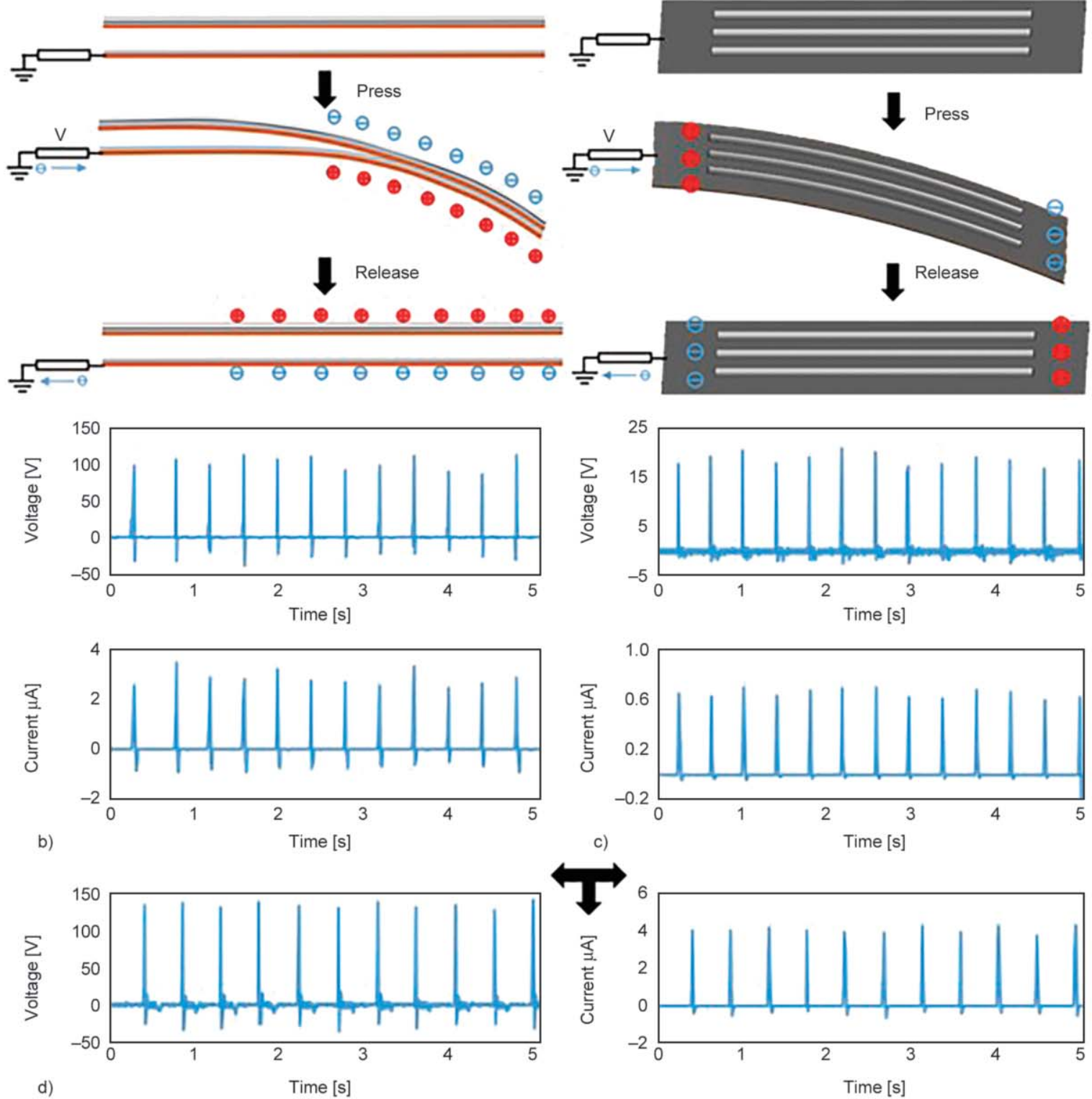

Figure 3. A fully packaged PTHG with TENG/PENG (a) the layer of piezoelectric NMFs deposited on the zinc plated layer and one pair of Cu-PCB TENG. Individually measured output voltage signals and the working principle of the (b) TENG (c) PENG respectively. (d) Electrical signals of the hybridization of PTHG. 


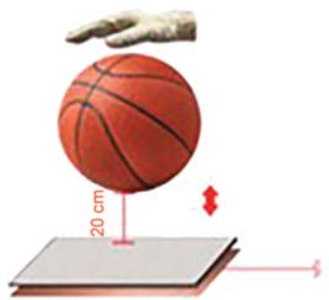

a)

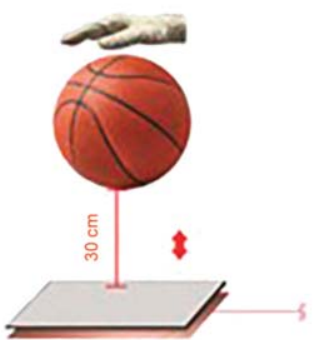

b)

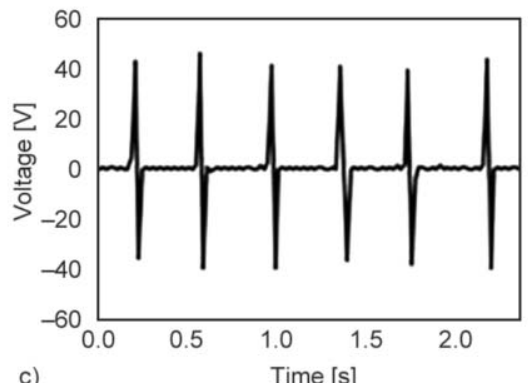

c)

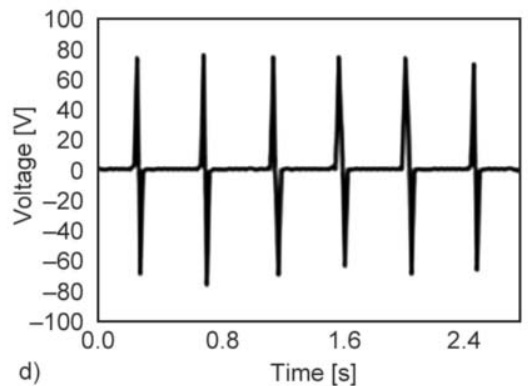

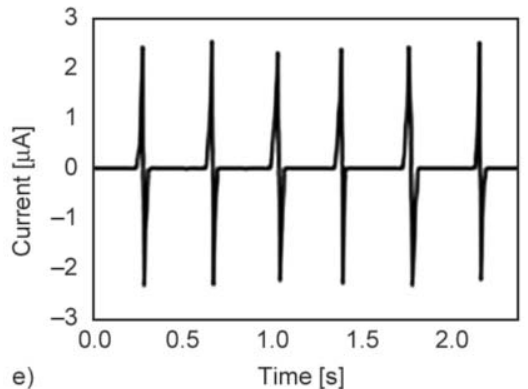

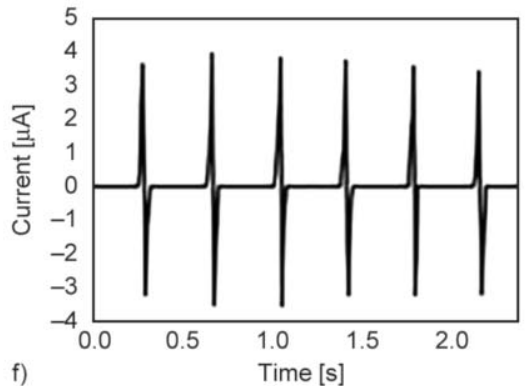

Figure 4. Illustration of PTHG fixed on table and operated by repeatedly hitting basketball on the device at two different separations of (a) $20 \mathrm{~cm}$ and (b) $30 \mathrm{~cm}$, respectively. The corresponding measured output voltage in (c-d) and output current in (e-f) respectively.

external resistance and the measured results indicated that the output voltage/current peak is about $18 \mathrm{~V} /$ $0.6 \mu \mathrm{A}$. Figure $3 \mathrm{~d}$ presents the hybridized device with the superimposed output voltage/current signals of $\sim 130 \mathrm{~V} / 4 \mu \mathrm{A}$ at $2 \mathrm{~Hz}$. The tested results show that both the PENG and TENG can be simultaneously operated in the synchronized way such that highly flexible hybridized self-powered sensor could be realized and outperformed the individually operated PENG or TENG. Validated tests such as polarity, superposition, Impedance matching were also performed to subsequently characterized the self-powered sensor as described before [39, 40].

In order to use the proposed stretchable PTHG for selfpowered detecting the repeatedly hitting basketball on the device at two different separations of Figure $4 \mathrm{a}$ $20 \mathrm{~cm}$ and Figure $4 \mathrm{~b} 30 \mathrm{~cm}$, respectively. As shown in Figure 4c, 4d for the integration of PTHG, the device could discernably distinguish the basketball patted from different heights $(20,30 \mathrm{~cm})$. Output voltage peak for 20 and $30 \mathrm{~cm}$ were measured as $\sim 40 \mathrm{~V} / 72 \mathrm{~V}$ respectively and the output current peak
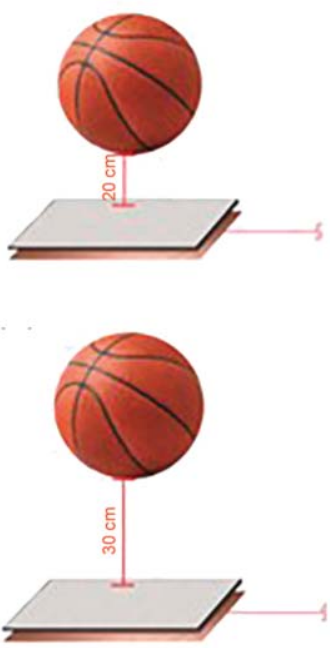
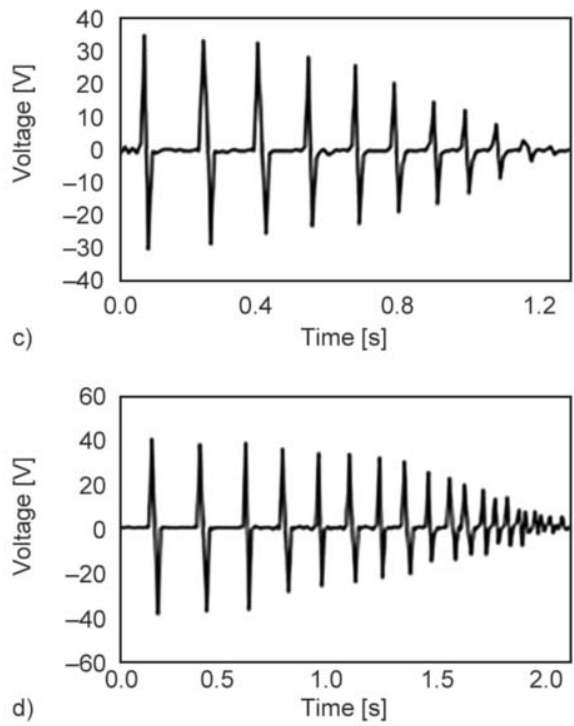

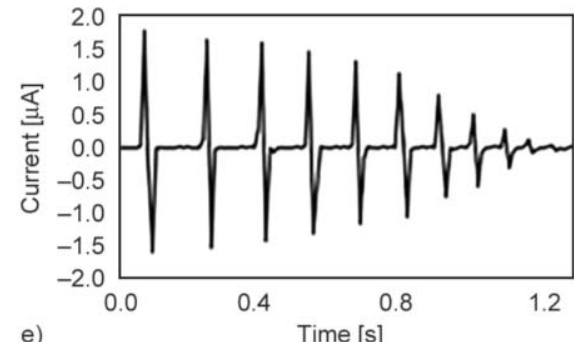

e)

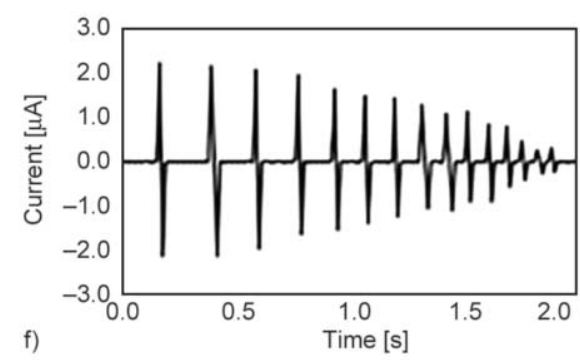

Figure 5. Performances of the ultrathin PTHG acted as an active sensor when fixed on table and operated by free fall basketball on the device at two different separations of (a) $20 \mathrm{~cm}$ and (b) $30 \mathrm{~cm}$ respectively, and corresponding electrical output for open-circuit (c-d) voltage and (e-f) current. 
were about $\sim 2.5 \mu \mathrm{A} / 3.5 \mu \mathrm{A}$ shown in Figure $4 \mathrm{e}, 4 \mathrm{f}$. Furthermore, in Figure 5a, 5b, the device detects and discriminates the damping behavior of a basketball freefall from different heights $(20,30 \mathrm{~cm})$. Output voltage under various height freefalling of 20 and $30 \mathrm{~cm}$ were measured as maximum output $\sim 33 \mathrm{~V} / 39 \mathrm{~V}$ respectively as shown in Figure 5c, 5d PTHG nonlinear output voltage resembles the shear
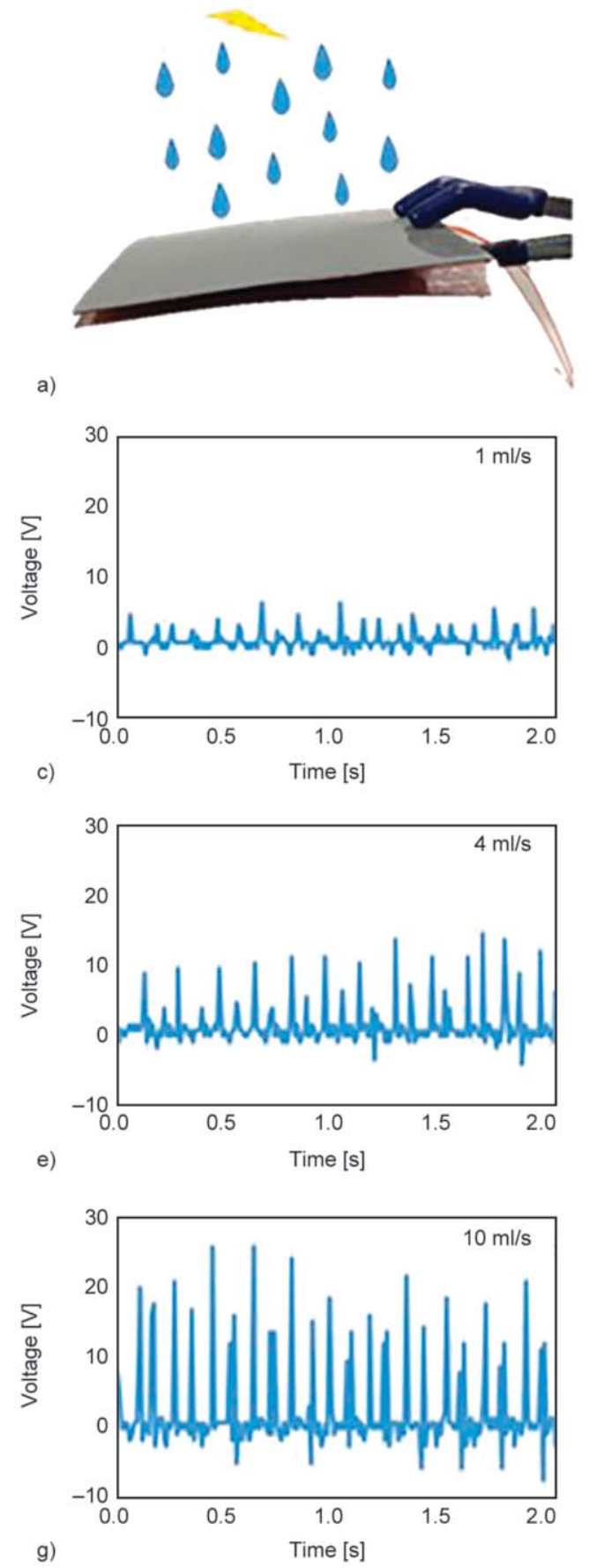

relaxation moduli of entangled comb polymers. In addition, in Figure 5e, 5f, the output maximum current of various freefalling height $(20,30 \mathrm{~cm})$ could be measured as $\sim 1.8 \mu \mathrm{A} / 2.2 \mu \mathrm{A}$ respectively. In addition, the measurement results agree well from both current and voltage results and the settling time for two different heights are measured as $1.2 \mathrm{~s} / 2.0 \mathrm{~s}$ respectively.
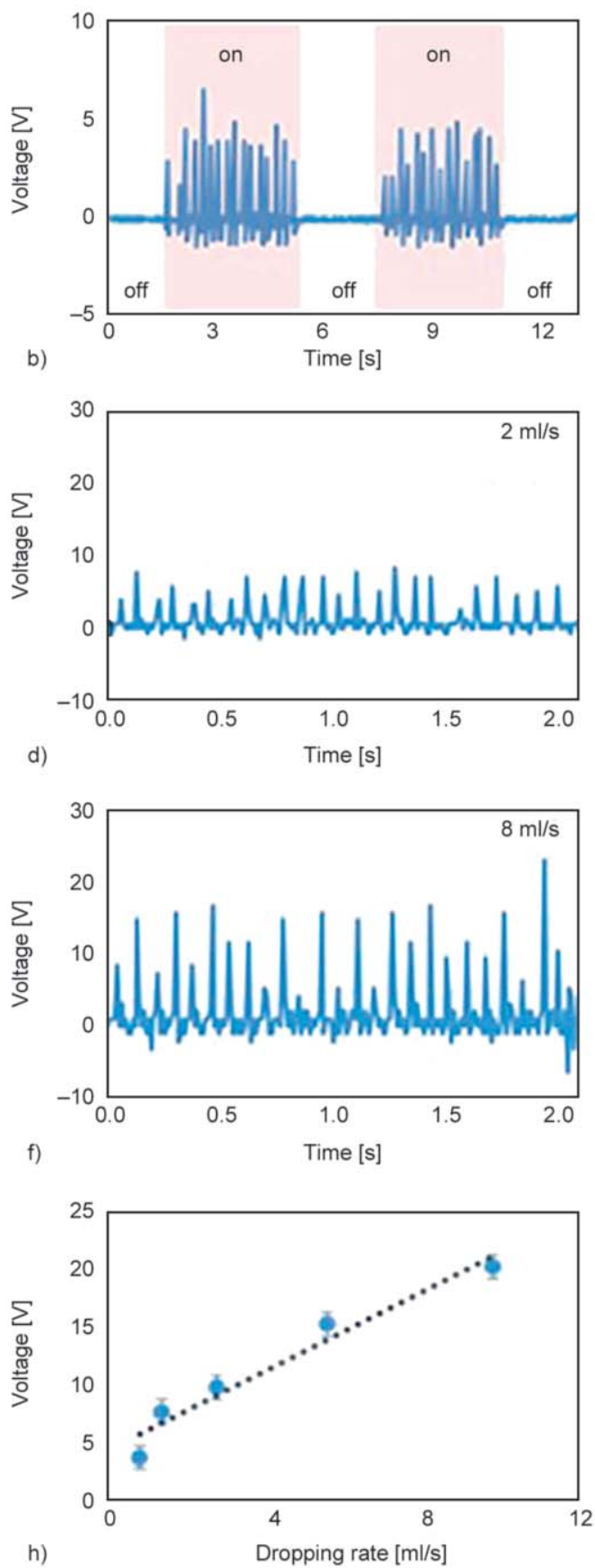

Figure 6. (a) Image of the PTHG fixed to holder and driven by simulated water dropping. Relative changes in voltage versus time for various water dropping rate. (b) Corresponding voltage output of the simulated water dropping in the on and off state. (c-g) Output voltage of device driven by different water dropping rate which are 1, 2, 4, 8, and $10 \mathrm{ml} / \mathrm{s}$ respectively. (h) The output voltage of PTHG is linearly increases from $3.8 \pm 0.43$ to $20.1 \pm 2.1 \mathrm{~V}$ with the input of simulated water dropping rate from 1 to $10 \mathrm{ml} / \mathrm{s}$. 
Energy transduction process can be simply illustrated as the gravitational potential energy of raindrops transferring into the mechanical energy to induce the piezoelectric/electrostatic energy via the proposed PTHG. Water drops were sprayed from a household showerhead to mechanically deform PTHG at a dropping rate of 1, 2, 4, 8, and $10 \mathrm{ml} / \mathrm{s}$, respectively, as shown in Figure $6 \mathrm{a}$. Figure $6 \mathrm{~b}$ shows the output voltage increases and reaches a maximum value of $\sim 4 \mathrm{~V}$ when the household shower was turned on at a dropping rate of $1 \mathrm{ml} / \mathrm{s}$. The measured voltage of the PTHG driven by household shower at different dropping rate is

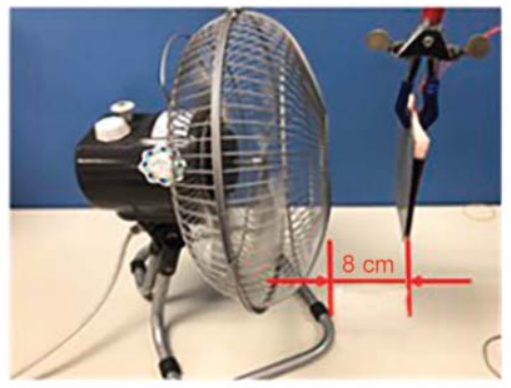

a)
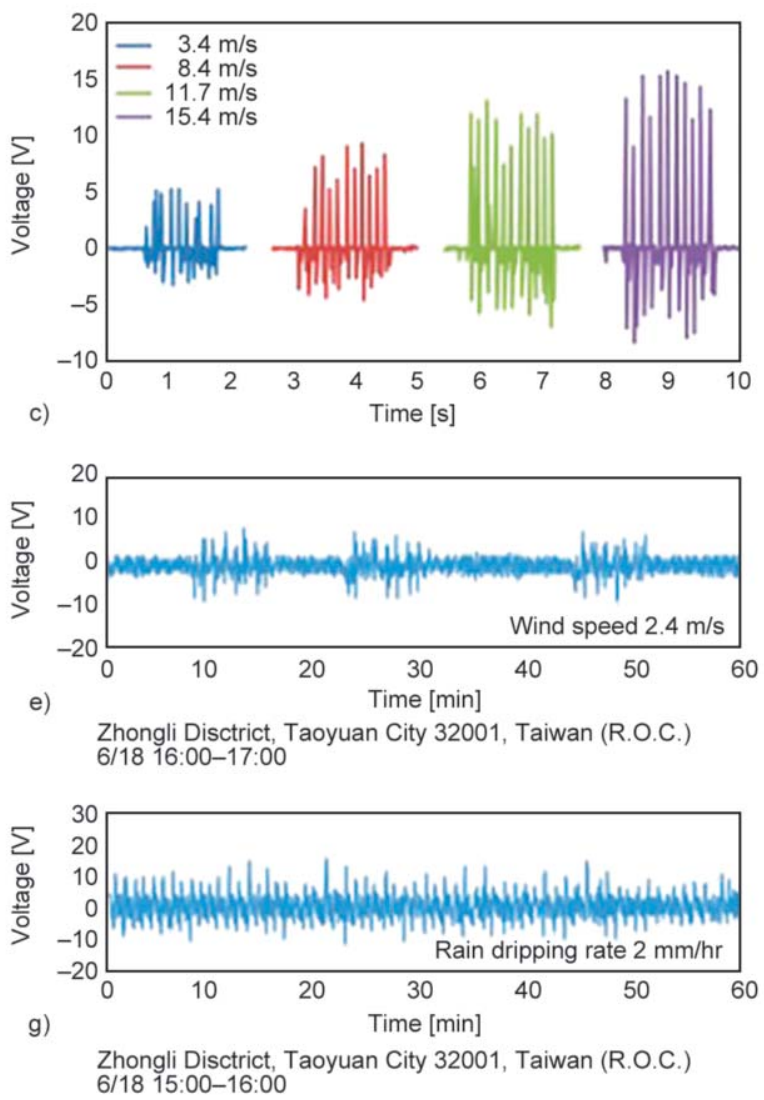

shown in Figure $6 \mathrm{c}-6 \mathrm{~g}$. The maximum output voltage reaches $\sim 20 \mathrm{~V}$ at a dropping rate of $10 \mathrm{ml} / \mathrm{s}$. With the increase of the water dropping rate, the output voltage values were proportionally increased. The dependences of output voltage of the PTHG on the water dropping rate as displayed in Figure 6h such that a self-powered rain drop monitoring sensor can be built with the positive slope of output voltage and the water dropping rate. Concerning the stability test, the PTHG output voltage recorded in three consecutive days and 15 minutes per day such that the device experimentally exhibits reliable consistence
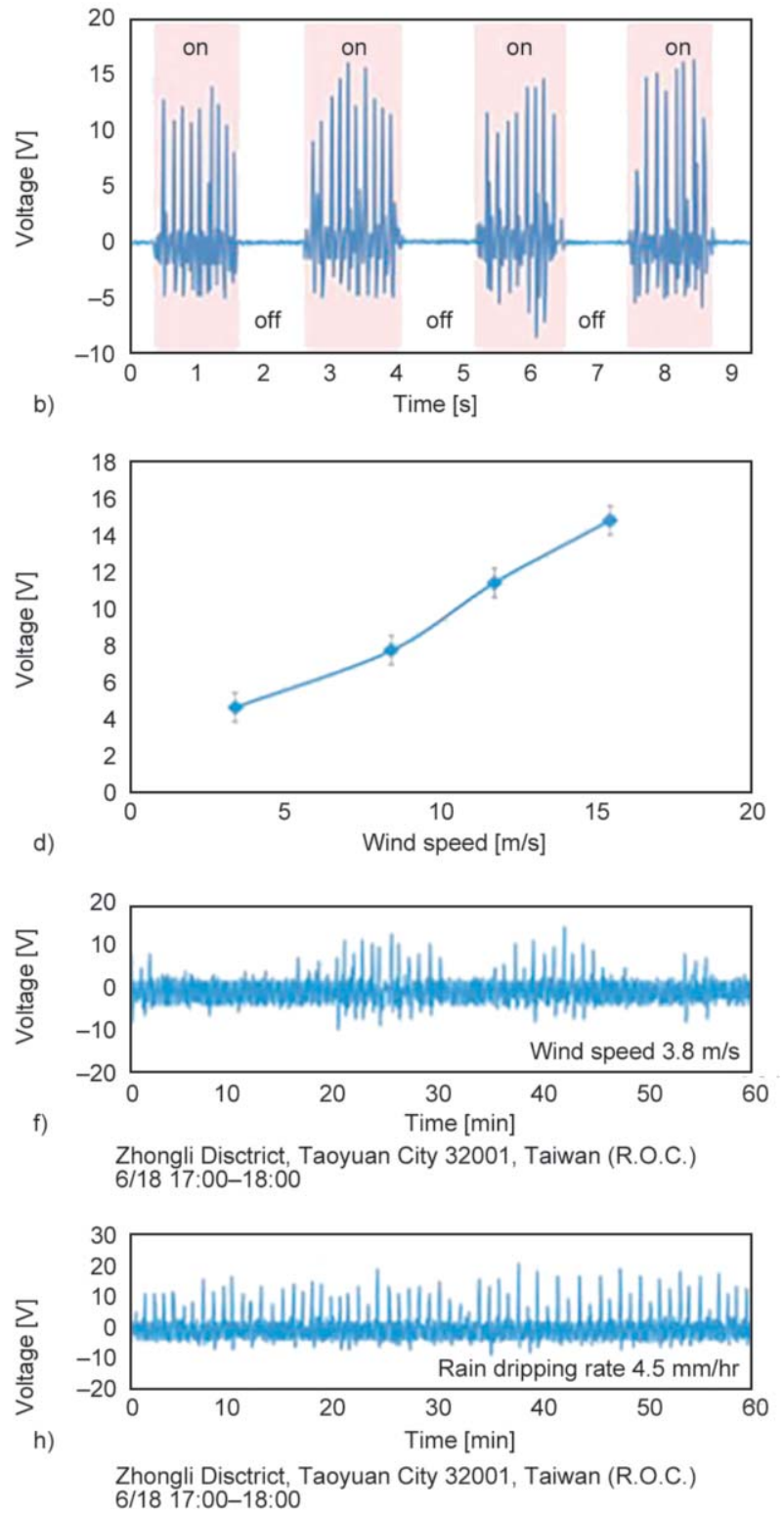

Figure 7. (a) Characterization of the PTHG induced from imitation of wind-speed measurement. (b) Corresponding voltage output of the electric fan in the on and off state. (c) Output voltages waveforms against flow velocities varying from 3.4 to $15.4 \mathrm{~m} / \mathrm{s}$. (d) Corresponding voltage output for wind speed from 3.4 to $15.4 \mathrm{~m} / \mathrm{s}$. Relative changes in voltage versus time for various wind blowing speed and rain dropping rate. (e) and (f) Output voltage of device driven by different wind speed which are $2.4 \mathrm{~m} / \mathrm{s}$ and $3.8 \mathrm{~m} / \mathrm{s}$. (g) and (h) Output voltage of device driven by different rain dropping rate which are $2 \mathrm{~mm} / \mathrm{hr}$ and $4.5 \mathrm{~mm} / \mathrm{hr}$, respectively. 
with a relatively negligible variation of peak output voltage, suggesting the robust power generating capability of the PTHG.

The experimental setup of the PTHG from imitation of natural wind-speed is schematically shown in Figure $7 \mathrm{a}$. Electric fan is placed in parallel to the PTHG at a distance of $\sim 8 \mathrm{~cm}$. In the experiment two positions of 'on' or 'off' state were manually controlled by turning the switch. As shown in Figure $7 \mathrm{~b}$, the PTHG output was less than $10 \mathrm{mV}$ during ' $v_{\text {off' }}$ ' state (primarily system noise) while the output voltage reaches an average value of 12 during 'on' state at the wind speed of $11.7 \mathrm{~m} / \mathrm{s}$. Another 'on' and 'off'cycle show repeatedly similar electrical output, indicating that the air-flow induced PTHG output was indeed from the mechanical interaction. The measured voltage of the PTHG for different wind speed is shown in Figure $7 \mathrm{c}$. For the wind speed of $3.4 \mathrm{~m} / \mathrm{s}$ to $15.4 \mathrm{~m} / \mathrm{s}$, the output maximum voltage reaches $\sim 15 \mathrm{~V}$. With the increase of the wind speed, the output voltage values were remarkably increased. The dependences of output voltage of the PTHG on the wind speed shown in Figure 7d. The device fixed the PTHG in holder and driven by natural wind blowing and rain dropping, as recorded at 2017/6/18 16:0017:00 and 17:00-18:00 in Zhongli District, Taoyuan City 32001, Taiwan (R.O.C.) respectively. Relative changes in voltage versus time for various wind blowing speed and rain dropping rate, which were given in detail in Figure 7e-7h, demonstrating the potential of developed device for harvesting the energy from the sustainable resources.

\section{Conclusions}

In summary, a hybridized power harvester was proposed to generate electricity from natural and sustainable resources such as raindrops and wind. The paper proposed a structural design of double plate configuration with nano-textured surface and piezoelectric fibers such that the piezoelectric and triboelectric generations could be integrated simultaneously. The PTHG is capable of individually/simultaneously converting mechanical energies from the environment into electricity. Bio-mechanical motions of basketball dribbling at different height of 20 and $30 \mathrm{~cm}$ can be discriminated in a self-powered manner. Output voltage under various patting height of 20 and $30 \mathrm{~cm}$ were measured as $\sim 40 \mathrm{~V} / 72 \mathrm{~V}$ respectively and the output current were about $\sim 2.5 \mu \mathrm{A} / 3.5 \mu \mathrm{A}$. The maximum area power density can be reached as $4.04 \mathrm{~mW} / \mathrm{m}^{2}$ for dribbling the basketball at height of $30 \mathrm{~cm}$. In addition, the energy harvesting capability of proposed PTHG can be driven by the water dropping rate of 1 to $10 \mathrm{ml} / \mathrm{s}$, the output maximum voltage reaches $\sim 20 \mathrm{~V}$ and area power density $\sim 0.981 \mathrm{~mW} / \mathrm{m}^{2}$. Driven by the wind speed of 3.4 to $15.4 \mathrm{~m} / \mathrm{s}$, the output maximum voltage reaches $\sim 15 \mathrm{~V}$ and $\sim 0.783 \mathrm{~mW} / \mathrm{m}^{2}$ area power density. The proposed PTHG can collectively generate the output voltage as driven by the natural wind speed from the environment in the range of 2.4 and $3.8 \mathrm{~m} / \mathrm{s}$ and actual precipitation ( 2 and $4.5 \mathrm{~mm} / \mathrm{hr}$ ) at $2017 / 6 / 18$ 16:00-17:00 and 17:00-18:00 in Zhongli District, Taoyuan City 32001, Taiwan (R.O.C.), as recorded by the central weather bureau, Taiwan.

\section{References}

[1] Meyar-Naimi H., Vaez-Zadeh S.: Sustainable development based energy policy making frameworks, A critical review. Energy Policy, 43, 351-361 (2012).

https://doi.org/10.1016/j.enpol.2012.01.012

[2] Paradiso J. A., Starner T.: Energy scavenging for mobile and wireless electronics. IEEE Pervasive Computing, 4, 18-27 (2005). https://doi.org/10.1109/MPRV.2005.9

[3] Wang Z. L., Song J.: Piezoelectric nanogenerators based on zinc oxide nanowire arrays. Science, 312, 242-246 (2006). https://doi.org/10.1126/science.1124005

[4] Zhu G., Chen J., Zhang T. J., Jing Q. S., Wang Z. L.: Radial-arrayed rotary electrification for high performance triboelectric generator. Nature Communications, 5, 3426/1-3426/9 (2014). https://doi.org/10.1038/ncomms4426

[5] Li X., Tao J., Wang X., Zhu J., Pan C., Wang Z. L.: Networks of high performance triboelectric nanogenerators based on liquid-solid interface contact electrification for harvesting low-frequency blue energy. Advanced Energy Materials, 8, 1800705/1-1800705/7 (2018). https://doi.org/10.1002/aenm.201800705

[6] Sato S., Yamada K., Inagaki N.: System for simultaneously monitoring heart and breathing rate in mice using a piezoelectric transducer. Medical and Biological Engineering and Computing, 44, 353-362 (2006). https://doi.org/10.1007/s11517-006-0047-z

[7] Kanik M., Say M.G., Daglar B., Yavuz A. F., Dolas M. H., El-Ashry M. M., Bayindir M.: A motion- and soundactivated, 3D-printed, chalcogenide-based triboelectric nanogenerator. Advanced Material, 27, 2367-2376 (2015). https://doi.org/10.1002/adma.201405944 
[8] Li Z., Wang Z. L.: Air/liquid-pressure and heartbeatdriven flexible fiber nanogenerators as a micro/nanopower source or diagnostic sensor. Advanced Material, 23, 84-89 (2011). https://doi.org/10.1002/adma.201003161

[9] Hwang B-U., Lee J-H., Trung T-Q., Roh E., Kim D-I., Kim S-W., Lee N-E.: Transparent stretchable self-powered patchable sensor platform with ultrasensitive recognition of human activities. ACS Nano, 9, 8801-8810 (2015). https://doi.org/10.1021/acsnano.5b01835

[10] Roundy S., Takahashi E.: A planar electromagnetic energy harvesting transducer using a multi-pole magnetic plate. Sensors and Actuators A: Physical, 195, 98-104 (2013). https://doi.org/10.1016/j.sna.2013.03.018

[11] Choi M-Y., Choi D., Jin M-J., Kim I., Kim S-H., Choi J-Y., Lee S. Y., Kim J. M., Kim S-W.: Mechanically powered transparent flexible charge-generating nanodevices with piezoelectric $\mathrm{ZnO}$ nanorods. Advanced Materials, 21, 2185-2189 (2009).

https://doi.org/10.1002/adma.200803605

[12] Gullapalli H., Vemuru V. S., Kumar A., Botello-Mendez A., Vajtai R., Terrones M., Nagarajaiah S., Ajayan P. M.: Flexible piezoelectric $\mathrm{ZnO}$-paper nanocomposite strain sensor. Small, 6, 1641-1646 (2010). https://doi.org/10.1002/smll.201000254

[13] Wang X., Wang S., Yang Y., Wang Z. L.: Hybridized electromagnetic-triboelectric nanogenerator for scavenging air-flow energy to sustainably power temperature sensors. ACS Nano, 9, 4553-4562 (2015). https://doi.org/10.1021/acsnano.5b01187

[14] Xu S., Hansen B. J., Wang Z. L.: Piezoelectric-nanowire-enabled power source for driving wireless microelectronics. Nature Communications, 1, 93/1-93/5 (2010). https://doi.org/10.1038/ncomms1098

[15] Lee J-H., Lee K. Y., Gupta M. K., Kim T. Y., Lee D-Y., Oh J., Ryu C., Yoo W. J., Kang C-Y., Yoon S-J., Yoo J-B., Kim S-W.: Highly stretchable piezoelectric-pyroelectric hybrid nanogenerator. Advanced Materials, 26, 765769 (2014). https://doi.org/10.1002/adma.201303570

[16] Zi Y., Lin L., Wang J., Wang S., Chen J., Fan X., Yang P-K., Yi F., Wang Z. L.: Triboelectric-pyroelectricpiezoelectric hybrid cell for high-efficiency energy-harvesting and self-powered sensing. Advanced Materials, 27, 2340-2347 (2015). https://doi.org/10.1002/adma.201500121

[17] Kumar B., Lee K. Y., Park H-K., Chae S. J., Lee Y. H., Kim S-W.: Controlled growth of semiconducting nanowire, nanowall, and hybrid nanostructures on graphene for piezoelectric nanogenerators. ACS Nano, 5, 4197 4204 (2011).

https://doi.org/10.1021/nn200942s
[18] Bae S-H., Kahya O., Sharma B. K., Kwon J., Cho H. J., Özyilmaz B., Ahn J-H.: Graphene-P(VDF-TrFE) multilayer film for flexible applications. ACS Nano, 7, 3130-3138 (2013). https://doi.org/10.1021/nn400848j

[19] Kwon J., Seung W., Sharma B. K., Kim S-W., Ahn J-H.: A high performance PZT ribbon-based nanogenerator using graphene transparent electrodes. Energy and Environmental Science, 5, 8970-8975 (2012). https://doi.org/10.1039/C2EE22251E

[20] Fuh Y-K., Chen P-C., Huang Z-M., Ho H-C.: Self-powered sensing elements based on direct-write, highly flexible piezoelectric polymeric nano/microfibers. Nano Energy, 11, 671-677 (2015). https://doi.org/10.1016/j.nanoen.2014.10.038

[21] Wang X., Song J., Liu J., Wang Z. L.: Direct-current nanogenerator driven by ultrasonic waves. Science, 316, 102-105 (2007). https://doi.org/10.1126/science.1139366

[22] Chang C., Tran V. H., Wang J., Fuh Y-K., Lin L.: Directwrite piezoelectric polymeric nanogenerator with high energy conversion efficiency. Nano Letters, 10, 726731 (2010). https://doi.org/10.1021/n19040719

[23] Fuh Y-K., Chen P-C., Ho H-C., Huang Z-M., Li S-C.: All-direction energy harvester based on nano/micro fibers as flexible and stretchable sensors for human motion detection. RSC Advances, 5, 67787-67794 (2015). https://doi.org/10.1039/C5RA00275C

[24] Fuh Y-K., Chen S-Y., Ye J-C.: Massively parallel aligned microfibers-based harvester deposited via in situ, oriented poled near-field electrospinning. Applied Physics Letters, 103, 033114/1-033114/4 (2013). https://doi.org/10.1063/1.4813909

[25] Fuh Y-K., Ye J-C., Chen P-C., Ho H-C., Huang Z-M.: Hybrid energy harvester consisting of piezoelectric fibers with largely enhanced $20 \mathrm{~V}$ for wearable and muscle-driven applications. ACS Applied Materials and Interfaces, 7, 16923-16931 (2015).

https://doi.org/10.1021/acsami.5b03955

[26] Liu G., Leng Q., Lian J., Guo H., Yi X., Hu C.: Notepadlike triboelectric generator for efficiently harvesting low-velocity motion energy by interconversion between kinetic energy and elastic potential energy. ACS Applied Materials and Interfaces, 7, 1275-1283 (2015). https://doi.org/10.1021/am507477y

[27] Yang J., Chen J., Yang Y., Zhang H., Yang W., Bai P., $\mathrm{Su}$ Y., Wang Z. L.: Broadband vibrational energy harvesting based on a triboelectric nanogenerator. Advanced Energy Materials, 4, 1301322/1-1301322/9 (2014). https://doi.org/10.1002/aenm.201301322

[28] Yang W., Chen J., Jing Q., Yang J., Wen X., Su Y., Zhu G., Bai P., Wang Z. L.: 3D stack integrated triboelectric nanogenerator for harvesting vibration energy. Advanced Functional Materials, 24, 4090-4096 (2014). https://doi.org/10.1002/adfm.201304211 
[29] Bae J., Lee J., Kim S., Ha J., Lee B-S., Park Y. J., Choong C., Kim J-B., Wang Z. L., Kim H-Y., Park J-J., Chung U-I.: Flutter-driven triboelectrification for harvesting wind energy. Nature Communications, 5, 4929/14929/9 (2014).

https://doi.org/10.1038/ncomms5929

[30] Chen J., Yang J., Li Z., Fan X., Zi Y., Jing Q., Guo H., Wen Z., Pradel K. C., Niu S., Wang Z. L.: Networks of triboelectric nanogenerators for harvesting water wave energy: A potential approach toward blue energy. ACS Nano, 9, 3324-3331 (2015).

https://doi.org/10.1021/acsnano.5b00534

[31] Yang Y., Zhang H., Liu R., Wen X., Hou T-C., Wang Z. L.: Fully enclosed triboelectric nanogenerators for applications in water and harsh environments. Advanced Energy Materials, 3, 1563-1568 (2013).

https://doi.org/10.1002/aenm.201300376

[32] Fan X., Chen J., Yang J., Bai P., Li Z., Wang Z. L.: U1trathin, rollable, paper-based triboelectric nanogenerator for acoustic energy harvesting and self-powered sound recording. ACS Nano, 9, 4236-4243 (2015). https://doi.org/10.1021/acsnano.5b00618

[33] Lin Z-H., Cheng G., Lee S., Wang Z. L.: Harvesting water drop energy by a sequential contact-electrification and electrostatic-induction process. Advanced Materials, 26, 4690-4696 (2014). https://doi.org/10.1002/adma.201400373

[34] Zhu G., Su Y., Bai P., Chen J., Jing Q., Yang W., Wang Z. L.: Harvesting water wave energy by asymmetric screening of electrostatic charges on a nanostructured hydrophobic thin-film surface. ACS Nano, 8, 60316037 (2014).

https://doi.org/10.1021/nn5012732

[35] Yang Y., Zhang H., Chen J., Jing Q., Zhou Y. S., Wen X., Wang Z. L.: Single-electrode-based sliding triboelectric nanogenerator for self-powered displacement vector sensor system. ACS Nano, 7, 7342-7351 (2013). https://doi.org/10.1021/nn403021m

[36] Kim S., Gupta M. K., Lee K. Y., Sohn A., Kim T. Y., Shin K-S., Kim D., Kim S. K., Lee K. H., Shin H-J., Kim D-W., Kim S-W.: Transparent flexible graphene triboelectric nanogenerators. Advanced Materials, 26, 3918-3925 (2014). https://doi.org/10.1002/adma.201400172

[37] Lee K. Y., Chun J., Lee J. H., Kim K. N., Kang N. R., Kim J. Y., Kim M. H., Shin K. S., Gupta M. K., Baik J. M., Kim S. W.: Hydrophobic sponge structure-based triboelectric nanogenerator. Advanced Materials, 26, 5037-5042 (2014). https://doi.org/10.1002/adma.201401184

[38] Zhong J., Zhong Q., Fan F., Zhang Y., Wang S., Hu B., Wang Z. L., Zhou J.: Finger typing driven triboelectric nanogenerator and its use for instantaneously lighting up LEDs. Nano Energy, 2, 491-497 (2013).

https://doi.org/10.1016/j.nanoen.2012.11.015
[39] Fuh Y-K., Li S-C., Chen C-Y.: Piezoelectrically and triboelectrically hybridized self-powered sensor with applications to smart window and human motion detection. APL Materials, 5, 074202/1-074202/9 (2017).

https://doi.org/10.1063/1.4978913

[40] Fuh Y-K., Li S-C., Chen C-Y., Tsai C-Y.: A fully packaged self-powered sensor based on near-field electrospun arrays of poly(vinylidene fluoride) nano/micro fibers. Express Polymer Letters, 12, 136-145 (2018). https://doi.org/10.3144/expresspolymlett.2018.12

[41] Zhang K., Wang X., Yang Y., Wang Z. L.: Hybridized electromagnetic-triboelectric nanogenerator for scavenging biomechanical energy for sustainably powering wearable electronics. ACS Nano, 9, 3521-3529 (2015). https://doi.org/10.1021/nn507455f

[42] Zhong X., Yang Y., Wang X., Wang Z. L.: Rotatingdisk-based hybridized electromagnetic-triboelectric nanogenerator for scavenging biomechanical energy as a mobile power source. Nano Energy, 13, 771-780 (2015). https://doi.org/10.1016/j.nanoen.2015.03.012

[43] Shao H., Cheng P., Chen R., Xie L., Sun N., Shen Q., Chen X., Zhu Q., Zhang Y., Liu Y., Wen Z., Sun X.: Triboelectric-electromagnetic hybrid generator for harvesting blue energy. Nano-Micro Letters, 10, 54-62 (2018). https://doi.org/10.1007/s40820-018-0207-3

[44] Shen J., Li Z., Yu J., Ding B.: Humidity-resisting triboelectric nanogenerator for high performance biomechanical energy harvesting. Nano Energy, 40, 282-288 (2017). https://doi.org/10.1016/j.nanoen.2017.08.035

[45] Li Z., Shen J., Abdalla I., Yu J., Ding B.: Nanofibrous membrane constructed wearable triboelectric nanogenerator for high performance biomechanical energy harvesting. Nano Energy, 36, 341-348 (2017). https://doi.org/10.1016/j.nanoen.2017.04.035

[46] Li Z., Zhu M., Qiu Q., Yu J., Ding B.: Multilayered fiber-based triboelectric nanogenerator with high performance for biomechanical energy harvesting. Nano Energy, 53, 726-733 (2018).

https://doi.org/10.1016/j.nanoen.2018.09.039

[47] Wang J., Li X., Zi Y., Wang S., Li Z., Zheng L., Yi F., Li S., Wang Z. L.: A flexible fiber-based supercapacitortriboelectric-nanogenerator power system for wearable electronics. Advanced Materials, 27, 4830-4836 (2015). https://doi.org/10.1002/adma.201501934

[48] Niu S., Liu Y., Chen X., Wang S., Zhou Y. S., Lin L., Xie Y., Wang Z. L.: Theory of freestanding triboelectric-layer-based nanogenerators. Nano Energy, 12, 760774 (2015). https://doi.org/10.1016/j.nanoen.2015.01.013

[49] Xu L., Pang Y., Zhang C., Jiang T., Chen X., Luo J., Tang W., Cao X., Wang Z. L.: Integrated triboelectric nanogenerator array based on air-driven membrane structures for water wave energy harvesting. Nano Energy, 31, 351-358 (2017).

https://doi.org/10.1016/j.nanoen.2016.11.037 